Potencial Farmacológico de Chalconas: Uma Breve Revisão

\author{
Ferreira, M. K. A.; Fontenelle, R. O. S.; Magalhães, F. E. A.; Bandeira, P. \\ N.; S.; Menezes, J. E. S. A.; dos Santos, H.*
}

Rev. Virtual Quim., 2018, 10 (5), 1455-1473. Data de publicação na Web: 5 de novembro de 2018

http://rvq.sbq.org.br

\title{
Chalcones Pharmacological Potential: A Brief Review
}

\begin{abstract}
Chalcones are naturally occurring aromatic ketones, consisting of an $\alpha, \beta$-unsaturated carbonyl system linking two aromatic rings. It is a class of open chain flavonoids, abundantly found in plants of the families Leguminosae, Compositae and Moraceae, and present in fruits, vegetables, grains, roots, flowers, teas, wines, products regularly used for human consumption. Research that investigates the properties of chalcones has shown a broad spectrum of biological activity, which has been reinforcing since the isolation, synthesis, identification and investigation of biological effects. This study was carried out based on the scientific articles published on chalcones, emphasizing the following pharmacological activities: antioxidant, antinociceptive, anti-inflammatory, anxiolytic and anticonvulsant compared to the period of the last 10 years. It was verified that the reported activities prove that chalcones and their derivatives are substances of great chemical-pharmacological interest.
\end{abstract}

Keywords: Chalcones; biological activities; flavonoids.

\section{Resumo}

Chalconas são cetonas aromáticas de ocorrência natural, caracterizadas por um sistema carbonílico $\alpha, \beta$-insaturado que une dois anéis aromáticos. Pertencentes a classe dos flavonoides de cadeia aberta, abundantemente encontradas em plantas das famílias Leguminosae, Compositae e Moraceae, estão presentes em frutas, vegetais, grãos, raízes, flores, chás, vinhos, produtos regularmente utilizados para consumo humano. Estudos têm demonstrado o grande potencial farmacológico das chalconas. Esta revisão foi realizada com base nos artigos científicos publicados sobre chalconas, enfatizando as seguintes atividades farmacológicas: antioxidante, antinociceptiva, anti-inflamatória, ansiolítica e anticonvulsivante em relação ao período dos últimos dez anos. Foi verificado que as atividades relatadas comprovam que as chalconas e seus derivados são substâncias de grande interesse químico-farmacológico.

Palavras-chave: Chalconas; atividades biológicas; fármacos.

\footnotetext{
* Universidade Estadual Vale do Acaraú, Curso de Química, Laboratório de Química de Produtos Naturais, Síntese e Biocatálise de Compostos Orgânicos-LBPNSB, Campus Betania, CEP 62040-370, Sobral-CE, Brasil.

Mhelciodossantos@gmail.com

DOI: 10.21577/1984-6835.20180099
} 


\section{Potencial Farmacológico de Chalconas: Uma Breve Revisão}

Maria K. A. Ferreira, ${ }^{a}$ Raquel O. S. Fontenelle, ${ }^{b}$ Francisco E. A. Magalhães, ${ }^{c}$ Paulo N. Bandeira, ${ }^{d}$ Jane E. Silva A. de Menezes, ${ }^{a}$ Hélcio S. dos Santos ${ }^{\mathrm{d}, *}$

a Universidade Estadual do Ceará, Centro de Ciências e Tecnologia (CCT), Campus do Itaperi, Laboratório de Química de Produtos Naturais-LQPN-S,CEP 60741-000, Fortaleza-CE, Brasil.

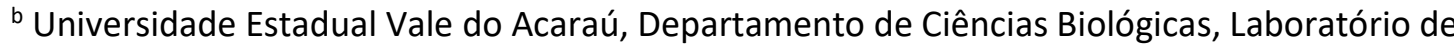
Microbiologia, Campus Betânia, CEP 62040-370, Sobral-CE, Brasil.

c Universidade Estadual do Ceará, Departamento de Química, Laboratório de Bioprospecção de Produtos Naturais e Biotecnologia, Campus CECITEC, CEP 60660-000, Tauá-CE, Brasil.

' Universidade Estadual Vale do Acaraú, Curso de Química, Laboratório de Química de Produtos Naturais, Síntese e Biocatálise de Compostos Orgânicos-LBPNSB, Campus Betania, CEP 62040370, Sobral-CE, Brasil.

* helciodossantos@gmail.com

Recebido em 18 de maio de 2018. Aceito para publicação em 22 de outubro de 2018

\section{Introdução}

\section{Metodologia}

3. Fontes Naturais e Sintéticas de Chalconas

\section{Atividades Farmacológicas de Chalconas}

4.1. Atividade antioxidante

4.2. Atividade antinociceptiva

4.3. Atividade anti-inflamatória

4.4. Atividade anticonvulsivante

4.5. Atividade antiacetilcolinesterase

\section{Considerações Finais}

\section{Introdução}

Chalconas são bioprecursoras dos flavonoides e apresentam uma estrutura básica de [1,3-difenil-2-propen-1-ona] (Figura 1) sendo, portanto, cetonas aromáticas que ocorrem naturalmente, consistindo de um sistema de carbonila $\alpha, \beta$-insaturado que une dois anéis aromáticos. Constituem uma classe de flavonoides de cadeia aberta, abundantemente encontradas em plantas das famílias Leguminosae, Compositae e Moraceae, presente em frutas, vegetais, grãos, raízes, flores, chás, vinhos, produtos 
estes regularmente utilizados para consumo humano. ${ }^{2}$ Nas flores, uma característica marcante é a sua capacidade de conferir pigmento amarelo às pétalas de algumas plantas de uso medicinal, auxiliando também na polinização como atraentes de insetos e/ou pássaros. ${ }^{1,2}$

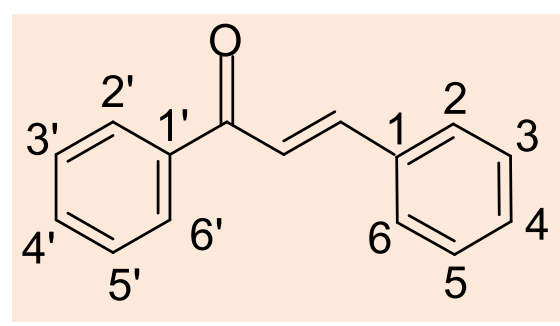

Figura 1. Estrutura básica das chalconas

Chalconas e seus derivados são substâncias de grande interesse químico-farmacológico e tem recebido uma grande atenção devido, sobretudo, a sua estrutura relativamente simples e a diversidade de atividades farmacológicas que apresentam, dentre as quais podemos citar: atividade antioxidante, ${ }^{3}$ antinociceptiva, ${ }^{4}$ anticonvulsivante, ${ }^{5}$ e antiinflamatória. $^{6}$

Os derivados 1,3-diaril-2-propen-1-onas obtidos através de plantas ou síntese orgânica têm sido amplamente explorados. Desta forma, muitas chalconas e seus análogos sintéticos têm demonstrado a capacidade de inibir a síntese de óxido nítrico (NO) e prostaglandinas (PG), que são produtos das vias óxido nítrico sintetase (NOS) e cicloxigenase (COX), sendo as principais vias pró-inflamatórias. Assim, compostos que atuam nessas vias se constituem promissores para o desenvolvimento de fármacos antiinflamatórios e antinociceptivos. ${ }^{7}$

Contudo, a inflamação é um processo biológico complexo que ameaça a saúde humana, de forma prolongada podendo causar várias doenças, como artrite, e até mesmo câncer. ${ }^{1}$ Portanto, os medicamentos mais utilizados no tratamento do processo inflamatório são os medicamentos antiinflamatórios não esteroides (AINEs), que representam cerca de $35 \%$ do mercado global de prescrição de medicamentos contra a dor.

Entretanto, os AINEs comuns, como aspirina (1) e indometacina(2) (Figura 2), podem inibir tanto a cicloxigenase-1 (COX-1) como a cicloxigenase-2 (COX-2) apresentando toxicidade significativa no trato gastrointestinal e renal. Por essa razão, justifica-se a necessidade de desenvolver novos fármacos anti-inflamatórios.

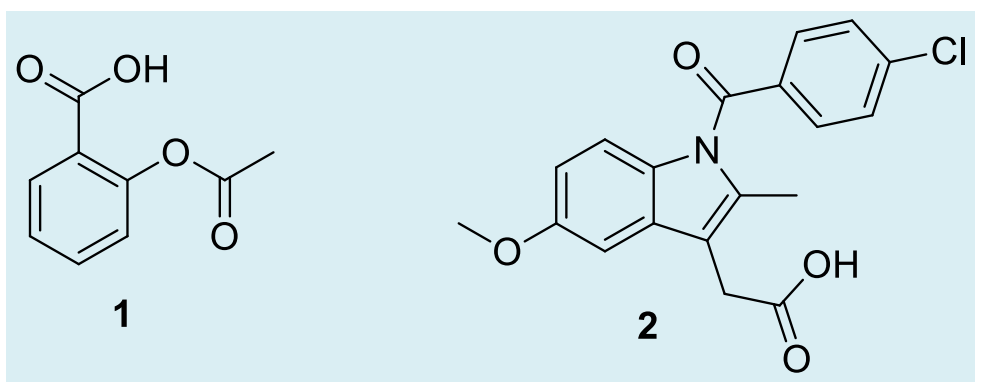

Figura 2. Representação estrutural da aspirina (1) e indometacina (2)

As chalconas de ocorrência natural, assim como seus análogos sintéticos, também demostraram outras importantes atividades biológicas e farmacológicas, incluindo 
potencial antioxidante e anticonvulsivante. A atividade antioxidante das chalconas pode ser justificada pela forte reatividade com grupos tiol em organismos vivos, pela indução de uma série de reações com radicais hidroxílicos em células vivas, ou pela redução dos mecanismos antioxidantes, como o teor de glutationa. Com base na investigação do potencial de atividade anticonvulsivante, alguns trabalhos empregam o modelo do pentilentetrazol (PTZ), método mais utilizado para a busca de substâncias com potencial efeito anticonvulsivante, incluindo os extratos de plantas. $^{8}$

Em vista das aplicações e propriedades das chalconas, assim como sua estrutura como ponto de partida para síntese de novos compostos derivados, esta revisão fornece um panorama geral da origem e as principais atividades biológicas com potencial farmacológico das chalconas.

\section{Metodologia}

A revisão foi realizada com base nos artigos científicos publicados sobre chalconas de origem natural e sintética, enfatizando as seguintes atividades farmacológicas: antioxidante, antinociceptiva, antiinflamatória, ansiolítica, anticonvulsivante e antiacetilcolinesterase em relação ao período dos últimos dez anos. Dessa forma, os bancos de dados utilizados foram: PubMed (http://www.ncbi.nlm.nih.gov/pubmed),

Science Direct

(http://www.sciencedirect.com/) e Google Scholar (https://scholar.google.com).

\section{Fontes Naturais e Sintéticas de Chalconas}

Flavonoides são compostos biossintéticos encontrados nas plantas, e possuem ação comprovada em pesquisas nas áreas da saúde, no combate a doenças. ${ }^{9-11}$ Dessa forma, o estudo dos mecanismos de biossíntese de compostos como os flavonoides torna-se indispensável para a obtenção de substâncias que possuam potencial terapêutico promissor. Portanto, na biossíntese de flavonoides, os isômeros chalcona (cis e trans), conhecidos como 1,3-difenil-2-propen1-ona, são intermediários comuns, considerados precursores (Esquema 1) encontrados em plantas de uso medicinal. ${ }^{12,13}$

Dessa maneira, as primeiras estruturas chalcônicas foram sintetizadas em laboratório em meados de 1800 , e seu isolamento de plantas ocorreu a partir de $1910 .{ }^{14}$ Além disso, estão presentes em várias plantas dicotiledôneas, algumas monocotiledôneas, pteridófitas e gimnospermas, mas são sintetizadas como componentes principais nas famílias Asteraceae, Moraceae, Fabaceaee Aristolochiaceae. ${ }^{15}$

O potencial biológico das chalconas vem sendo explorado desde a década de 40 , mas somente na década de 70 pesquisadores tiveram maior interesse pelo fato das chalconas terem demonstrado atividades antitumoral e antiparasitária. ${ }^{12}$ Assim, as chalconas sintéticas têm sido exploradas devido à flexibilidade na sua obtenção. Um grande número de rotas sintéticas foi relatado para a síntese de chalconas, sendo a mais clássica e geral a condensação de ClaisenSchmidt em meio básico (Esquema 2) ${ }^{16}$

Os membros do gênero Bidens e Coreopsis da família Asteraceae são conhecidos por conter alto teor de chalconas. Foram encontradas nas raízes aéreas de Ficus microcarpa, raízes, caules e folhas do gênero Morus, folhas e/ou frutos do gênero Artocarpus e membros do gênero Dorstenia, todos pertencentes à família Moraceae. ${ }^{18-20}$ $\mathrm{Na}$ família Fabaceae, as chalconas estão presentes em Desmodium renifolium, Psoralea corylifolia, raízes do gênero Sophora, Glycyrrhiza e no gênero Dalbergia. ${ }^{21}$ As chalconas também estão presentes em espécies de plantas de outras famílias, às vezes, em concentrações muito altas, como na seiva amarela da Angelica keiske ${ }^{22}$ ou em membros do gênero Scutellaria. ${ }^{17}$ 


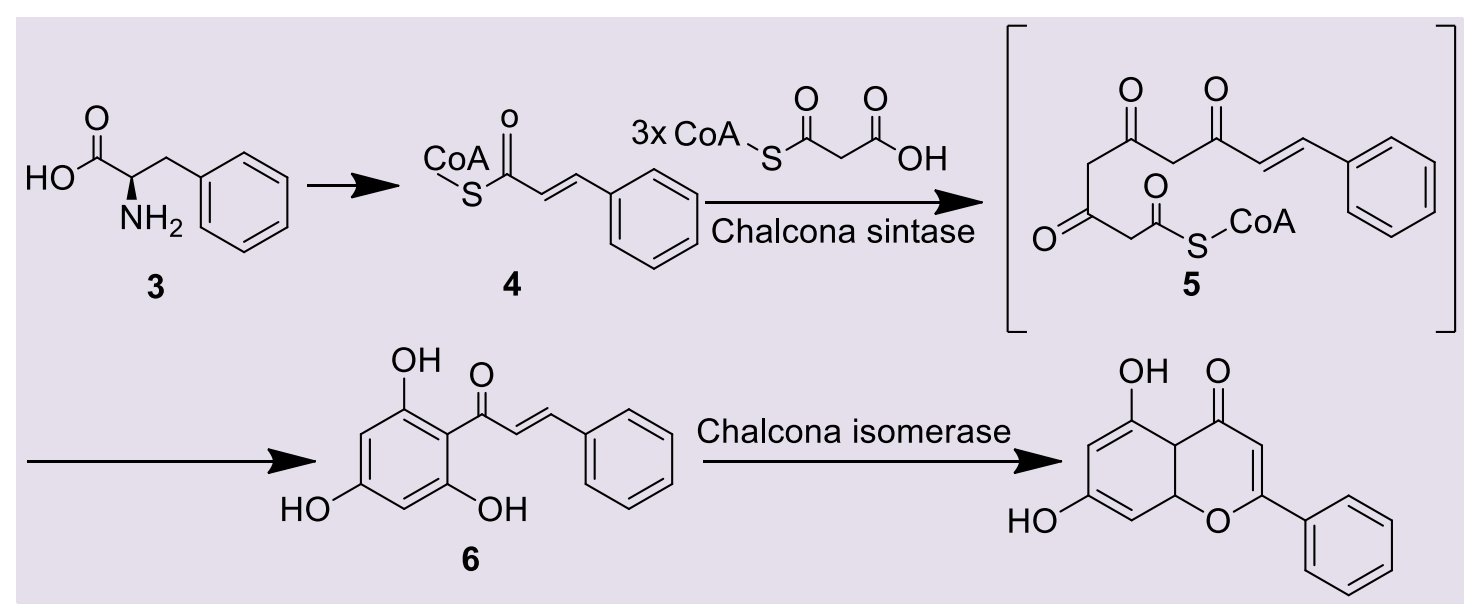

Esquema 1. Via biossintética dos flavonoides. $\mathbf{3}=$ L-fenilalanina; $\mathbf{4}=$ Cinamoil-CoA; $\mathbf{5}=$ MalonilCoA; $6=$ Chalcona hidroxilada

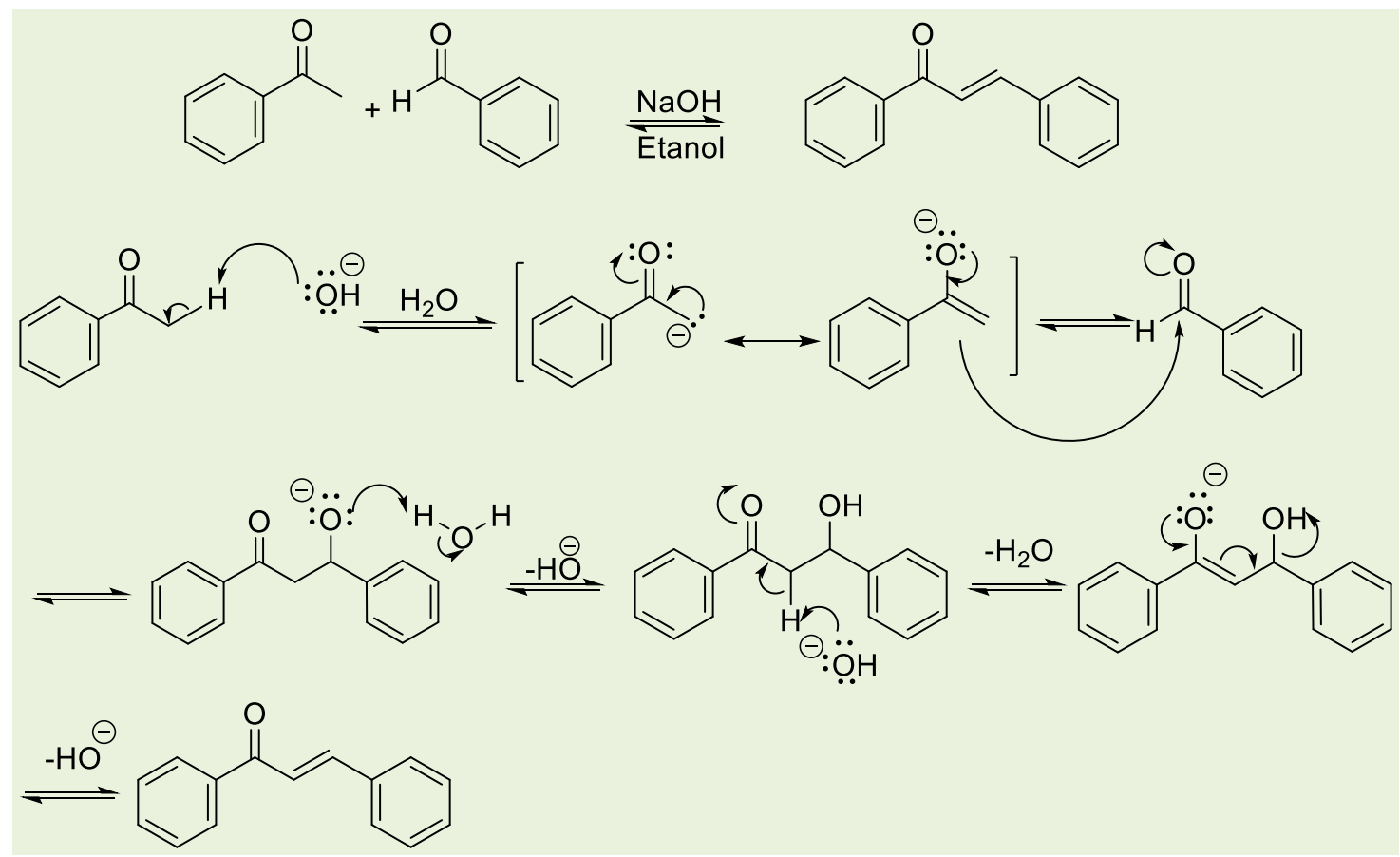

Esquema 2. Mecanismo da reação de condensação de Claisen-Schmidt

Os membros do gênero Bidens e Coreopsis da família Asteraceae são conhecidos por conter alto teor de chalconas. Foram encontradas nas raízes aéreas de Ficus microcarpa, raízes, caules e folhas do gênero Morus, folhas e/ou frutos do gênero Artocarpus e membros do gênero Dorstenia, todos pertencentes à família Moraceae. ${ }^{18-20}$ $\mathrm{Na}$ família Fabaceae, as chalconas estão presentes em Desmodium renifolium, Psoralea corylifolia, raízes do gênero Sophora, Glycyrrhiza e no gênero Dalbergia. ${ }^{21}$ As chalconas também estão presentes em espécies de plantas de outras famílias, às vezes, em concentrações muito altas, como na seiva amarela da Angelica keiske ${ }^{22}$ ou em membros do gênero Scutellaria. ${ }^{17}$ 


\section{Atividades Farmacológicas de Chalconas}

\subsection{Atividade antioxidante}

Os flavonoides são conhecidos pela sua potente capacidade antioxidante, capaz de eliminar radicais livres. Alguns estudos demonstraram que estes possuem efeitos protetores contra muitas doenças infecciosas e degenerativas, assim como doenças bacterianas e virais, cardiovasculares, cânceres e outras doenças relacionadas à idade. Parte dessas atividades estão relacionadas, pelo menos parcialmente, com sua capacidade de eliminação de radicais livres. ${ }^{23}$ Portanto, os radicais livres são gerados pelo nosso corpo por vários sistemas endógenos, quando expostos a diferentes condições físico-químicas ou estados patológicos, sendo um equilíbrio entre radicais livres e antioxidantes, condição necessária para uma função fisiológica adequada. ${ }^{23}$
Nas chalconas, as propriedades antioxidantes são influenciadas pela presença de determinados substituintes na molécula. Assim, o substituinte hidroxila passa a ser um dos responsáveis pelo aumento considerável da atividade antioxidante da chalcona como resultado da sua conversão para os radicais fenoxi correspondentes através do mecanismo de transferência de átomos de hidrogênio. $^{24}$

Recentemente, Vanangamudi e colaboradores $^{3}$ analisaram a atividade antioxidante de chalconas sintetizadas por condensação de Claisen-Schmidt. Caracterizadas por suas constantes físicas e dados espectrais, essas chalconas foram analisadas também quanto à atividade antibacteriana, antifúngica, antioxidante e antifedante de insetos. As atividades antioxidantes de todas as chalconas sintetizadas foram avaliadas pelo efeito de eliminação do radical DPPH. Os resultados demonstraram que as chalconas (7-8) apresentaram promissora atividade antioxidante (Figura 3).

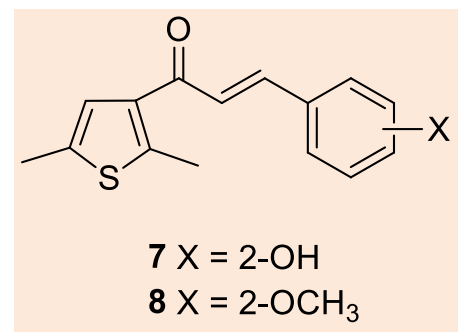

Figura 3. Estrutura das chalconas de 2,5-dimetil-3-tienil

Esta atividade foi justificada pela presença de grupos funcionais polares, fração hidrofóbica e a ausência de impedimento estérico perto do gupo aril, considerados fatores importantes para a presença de atividades biológicas de chalconas e seus derivados.

Cheng e colaboradores avaliaram o potencial antioxidante de broussochalcona $A$ (BCA) isolada de Broussonetiapapyrifera Vent., e seus efeitos na produção de óxido nítrico nos lipopolissacarídeos. Contudo, também exibiu eliminação de radicais livres, incluindo a inibição de superóxido e radicais hidroxila, o que pode contribuir para a sua peroxidação antilipídica em homogeneizados cerebrais de ratos. Em um sistema de ensaio de 2,2 difenil-1-picrilhidrazil, a atividade de eliminação de radicais do BCA mostrou-se mais potente do que a do $\alpha$-tocoferol, apresentando concentração inibitória (IC) de $7,6 \pm 0,8 \mu \mathrm{M}$. Dessa forma, estes resultados 
indicaram que o BCA (9) (Figura 4) é um poderoso antioxidante com atividade versátil de eliminação de radicais livres. ${ }^{25}$

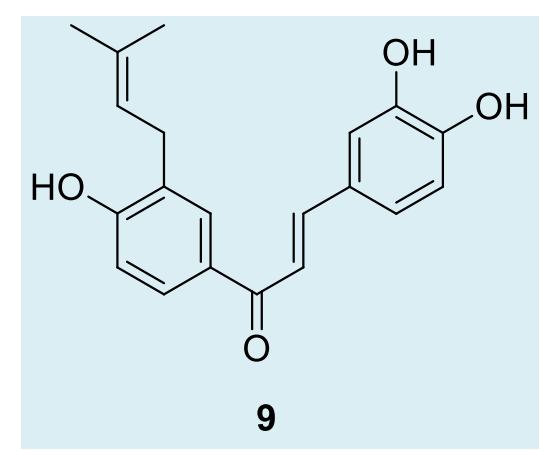

Figura 4. Estrutura da broussochalcona $A$

Shenvi e colaboradores sintetizaram uma série de 2,4,5-trimetoxi chalconas e observaram que, quando testadas contra três linhagens de células tumorais humanas (MCF7, SW-982 e HeLa) utilizando o ensaio MTT, revelaram que chalconas com grupos doadores de elétrons na posição para do anel aromático A (ligado ao grupo carbonila) demonstraram melhor atividade inibitória.
Com relação à atividade antioxidante, o composto (E)-1-(2,5-di-hidroxifenil)-3-(2,4,5trimetoxifenil)prop-2-en-1-ona (10) (Figura 5) mostrou atividade superior em relação a outras moléculas no ensaio de DPPH, indicando uma melhor capacidade de eliminação de radicais livres no ensaio de 2,2difenil-1-picrilhidrazil (DPPH). ${ }^{26}$<smiles>COc1cc(OC)c(OC)cc1/C=C/C(=O)c1c(O)cccc1O</smiles>

Figura 5. Representação estrutural da chalcona (E) -1-(2,5-dihidroxifenil)-3-(2,4,5trimetoxifenil)prop-2-en-1-ona

As propriedades antioxidantes das chalconas são bastante influenciadas pela presença de dois grupos arila e seus padrões de substituição, sendo o grupamento hidroxila um dos grupos-chave para aumentar a atividade antioxidante devido à sua fácil conversão ao radical fenoxi através do mecanismo de transferência de hidrogênio. ${ }^{26}$

De acordo com estudos relatados sobre as propriedades antioxidantes das chalconas no contexto da inflamação, Prabhakar e colaboradores realizaram uma investigação preliminar na busca de agentes antiinflamatórios com boas propriedades antioxidantes. Eles utilizaram uma biblioteca de dez membros formados por nove derivados monossubstituídos e uma chalcona não substituída. O procedimento metodológico ocorreu por meio de quatro ensaios in vitro bem estabelecidos, incluindo os ensaios de eliminação de radiação de peróxido de hidrogênio, óxido nítrico e superóxido, além da redução do ensaio de potência. Contudo, foi verificado que todos os derivados, exceto a 4'-fenilchalcona, apresentaram perfis 
antioxidantes em comparação com o composto original não substituído. ${ }^{27}$

O ensaio de potência redutora, revelou que a 2'-hidroxichalcona (11) (Figura 6) apresentou promissora atividade antioxidante $\left(\mathrm{IC}_{50}=25 \mu \mathrm{g} \mathrm{ml}^{-1}\right)$, quando comparado com o controle positivo ácido ascórbico. A atividade antioxidante in vitro dos derivados chalcônicos sintéticos (20 $\left.\mu \mathrm{g} \mathrm{ml} \mathrm{ml}^{-1}\right)$ foram comparados com os resultados obtidos da atividade anti-inflamatória (padrão ibuprofeno). Por conseguinte, foi observado que os compostos que se apresentaram com maiores ou menores ações anti-inflamatórias e antioxidantes revelaram-se idênticos em ambos os casos. No caso da 2-hidroxichalcona a presença do grupo hidroxila no anel A, contribuiu de maneira decisiva tanto para atividade antioxidante como para atividade anti-inflamatória.<smiles>O=C(/C=C/c1ccccc1)c1ccccc1O</smiles>

Figura 6. Estrutura da 2-hidroxichalcona

Lahsasni e colaboradores realizaram uma investigação acerca da atividade antioxidante de chalconas sintetizadas por meio da reação das mesmas com o ácido palmítico ou esteárico. Todos os compostos sintetizados foram avaliados com relação a sua capacidade antioxidante para reduzir a 2,2-difenil-1- picrilhidrazil (DPPH). Em particular, o composto (E)-3-(3-(4-nitrofenil)-3-oxoprop-1en-1-il)fenilpalmitato (12) (Figura 7), o qual apresentou $68,58 \%$ de inibição em [C] $=2 \mu \mathrm{g}$ $\mathrm{ml}^{-1}$. A atividade desses compostos foi muito próximo da atividade do ácido ascórbico, particularmente em baixas concentrações. ${ }^{24}$

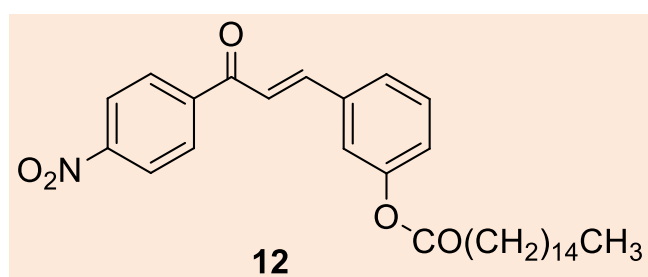

Figura 7. Estrutura da chalcona (E)-3-(3-(4-nitrofenil)-3-oxoprop-1-en-1-il)fenil palmitato

\subsection{Atividade antinociceptiva}

De acordo com a Associação Internacional para o Estudo da Dor (IASP), nocicepção é definido como "os processos neurais de codificação e processamento de estímulos nocivos", enquanto que a dor é relatada como sendo uma experiência desagradável sensorial e emocional associada a uma lesão tecidual real ou potencial ou descrita em termos de tal lesão. ${ }^{28}$
Nociceptores são terminações nervosas livres, não especializadas, que respondem a estímulos nociceptivos, detectando, desse modo, lesão nos tecidos, onde os estímulos desencadeados podem ser mecânicos, térmicos ou químicos. ${ }^{29}$ Assim, o potencial antinociceptivo de um produto natural ou sintético como chalconas, por exemplo, pode ser medido pelo seu poder de aumentar o limiar de excitação dessas terminações nervosas ao estímulo doloroso, ou então, fazer com que os nociceptores não percebam 
ou não respondam ao estímulo doloroso promovido. $^{28}$

Verificou-se que um número de chalconas e seus derivados inibem a síntese de óxido nítrico (NO) e prostaglandinas (PG), que são produtos das vias óxido nítrico sintetase (NOS) e cicloxigenase (COX). Essas vias perfazem as principais vias pró-inflamatórias e são direcionadas em muitas pesquisas para o desenvolvimento de fármacos antiinflamatórios e antinociceptivos. ${ }^{30}$

Estudos realizados por Mohamad e colaboradores examinaram a potencial atividade antinociceptiva da 6'-hidroxi-2',4'dimetoxichalcona (flavokawina B) (13) (Figura 8), uma chalcona extraída das raízes de Piper methysticum. Usando modelos de nocicepção química e térmica induzida em camundongos. Foi demonstrado que a flavokawina B (FKB, 0,$3 ; 1 ; 3$ ou $10 \mathrm{mg} / \mathrm{Kg}$ ) administrada por via oral (v.o) e intraperitoneal (i.p) produziu inibição significativa e dose dependente nas contrações abdominais induzidas pelo ácido acético (i.p), produzindo uma antinocicepção de aproximadamente 7 vezes mais potente do que a v.o. ${ }^{4}$

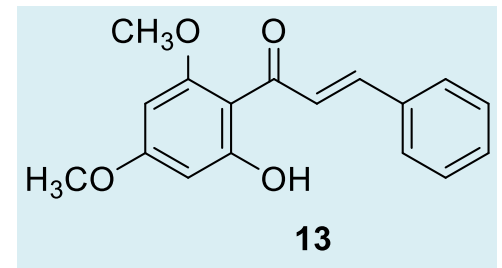

Figura 8: Representação estrutural da flavokawina B

Também foi demonstrado que o FKB produziu inibição significativa nas duas fases do teste de nocicepção (lamber a pata) induzida por formalina. O mesmo tratamento de flavokawina B (FKB) exibiu uma inibição significativa da nocicepção neurogênica induzida por injeções intraplantar de glutamato e capsaicina. Este composto também induziu um aumento significativo no período da resposta para estímulos térmicos, no teste da placa quente e seu efeito antinociceptivo não estava relacionado ao relaxante muscular ou ação sedativa. Além disso, o efeito antinociceptivo do FKB no teste de lamber a pata induzida por formalina e no teste de placa quente não foi afetado pelo pré-tratamento do antagonista não seletivo dos receptores opióides, a naloxona. Dessa forma, os resultados indicaram que FKB produziu um efeito antinociceptivo pronunciado contra modelos térmicos de dor em camundongos que exibiam atividade analgésica periférica e central. ${ }^{4}$
Ismail e colaboradores ${ }^{31}$ investigaram os efeitos antinociceptivos produzidos pela administração intraperitoneal de uma nova chalcona sintética (E)-1-(furan-2-il)-3-(2,3dimetoxifenil)prop-2-en-1-ona (DMFP) (14) (Figura 9). A administração de DMFP $(0,1 ; 0,5$; 1,0 ou $5,0 \mathrm{mg} / \mathrm{Kg}$ ) produziu uma atenuação significativa no teste de contorção abdominal induzido por ácido acético. Além disso, foi demonstrado que o DMFP exibiu uma inibição significativa da resposta nociceptiva neurogênica induzida por injeção intraplantar de capsaicina e glutamato. $O$ efeito antinociceptivo do DMFP no teste de contusão abdominal induzido pelo ácido acético e no teste de placa quente não foi antagonizado por pré-tratamento com um antagonista de receptor opioide não seletivo, a naloxona. Finalmente, o DMFP não mostrou efeitos tóxicos e/ou mortalidade num estudo de toxicidade aguda e não interferiu com a coordenação motora durante o teste rota-rod. 


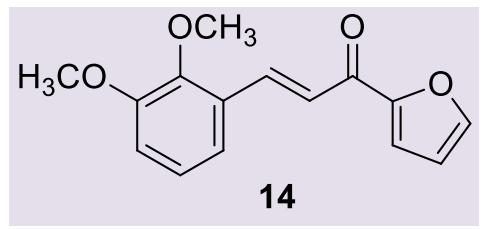

Figura 9. Fórmula estrutural da (E)-1-(furan-2-il)-3-(2,3-dimetoxifenil)prop-2-en-1-ona

Os resultados demostraram que o DMFP é responsável pela atividade antinociceptiva periférica, a qual está associada à produção atenuada e/ou liberação de NO e vários mediadores pró-inflamatórios, enquanto a atividade antinociceptiva central parece não estar relacionada ao sistema opioidérgico, mas pode envolver, pelo menos em parte, uma interação com a inibição de fibras sensíveis à capsaicina e o sistema glutamatérgico. ${ }^{31}$

Corrêa e colaboradores também avaliaram a atividade antinociceptiva em relação ao teste de contorção abdominal em camundongos de onze compostos do tipo chalcona, obtidos pela condensação de Claisen-Schmidt, utilizando diferentes aldeídos e acetofenonas substituídas. Alguns compostos, administrados por via intraperitoneal, causaram potente antinocicepção relacionada à dose, sendo várias vezes mais ativo do que alguns medicamentos de referência. Os compostos sintetizados mostraram um efeito antinociceptivo significativo em comparação com fármacos não esteroides, como aspirina, paracetamol e diclofenaco, com base no modelo de contorção abdominal induzida pelo ácido acético. Todos os compostos sintetizados foram mais potentes que a aspirina e o paracetamol; como por exemplo, o 3,4-diclorochalcona (15) (Figura 10) $(10,80$ $\mu \mathrm{M} \mathrm{Kg}{ }^{-1}$ ) foi dez vezes mais potente do que esses dois medicamentos padrão. ${ }^{32}$<smiles>O=C(/C=C/c1ccccn1)c1ccc(Cl)c(Cl)c1</smiles>

Figura 10. Fórmula estrutural da 3,4-diclorochalcona

Os resultados evidenciaram que alguns parâmetros físico-químicos estão envolvidos na atividade farmacológica. Isto porque, em geral, a introdução de grupos substituintes no anel $\mathrm{A}$ das chalconas produz compostos mais ativos.

\subsection{Atividade anti-inflamatória}

A investigação das atividades analgésicas e anti-inflamatórias é importante para o desenvolvimento de drogas a partir de plantas medicinais, uma vez que dor e inflamação são comumente observadas, ao mesmo tempo, nas doenças inflamatórias. As drogas antiinflamatórias são atualmente disponíveis para o tratamento das várias desordens inflamatórias, possuem um ou mais efeitos adversos e indesejáveis, efeitos colaterais, segundo Geetha e colaboradores. ${ }^{33}$ Assim, uma série de chalconas naturais e sintéticas foram avaliadas quanto ao seu possível papel como agentes anti-inflamatórios. A inflamação é uma complexa reação do corpo que expressa a resposta ao dano de suas células e tecidos vascularizados. Portanto, fazse necessário a busca por novas alternativas eficazes no tratamento dessa patologia. 
Viana e colaboradores ${ }^{34}$ apresentaram respostas positivas na investigação de atividades analgésicas e anti-inflamatórias de uma fração enriquecida em três chalconas diméricas (CD), isoladas com acetato de etila da casca do caule da espécie Myracrodruon urundeuva (Anacardiaceae), uma planta utilizada amplamente no Nordeste do Brasil, principalmente como um anti-inflamatório tópico do trato genital feminino. Dessa forma, observou-se que CD (5 e $10 \mathrm{mg} \mathrm{Kg}^{-1}$ ) inibiu as contorções abdominais em camundongos induzidas por ácido acético. No teste de formalina, $\mathrm{CD}$ (5 e $10 \mathrm{mg} \mathrm{Kg}^{-1}$ ) foi mais eficaz intraperitonealmente e inibiu predominantemente a segunda fase do teste. CD (10 e $20 \mathrm{Kg}^{-1}$; i.p) também aumentou o tempo de reação aos estímulos térmicos no teste da placa quente frente a ratos. No teste de edema da pata induzido por carragenina em ratos, CD (20 e $40 \mathrm{mg} \mathrm{Kg}^{-1}$; i.p) diminuiu significativamente o tamanho do edema de pata, após injeção de carragenina. CD (40 mg $\mathrm{Kg}^{-1} ;$ v.o) também se mostrou ativo. ${ }^{34}$

Em atribuição aos resultados anteriores, Botelho e colaboradores ${ }^{35}$ confirmaram que alguns estudos vêm sendo realizados validando os efeitos anti-inflamatórios e analgésicos de taninos e chalconas enriquecidas em frações (CEF) isoladas de plantas em modelos experimentais de inflamação, como o edema de pata induzido por carragenina, migração de neutrófilos em cavidades peritoneais e cistites hemorrágicas induzidas pela ciclofosfamida.

Derivados da chalcona 1,3difenilpropanona foram submetidos a rastreio anti-inflamatório usando o modelo de edema da pata do rato induzido por carragenina, isto porque os derivados de chalcona contêm uma porção de carbonila $\alpha, \beta$ insaturada que é responsável pela atividade anti-inflamatória. Os resultados mostraram que todos os derivados de chalcona apresentaram atividade anti-inflamatória com valor estatístico significativo de $P>0.05$. Contudo, os compostos 4-metóxi chalcona (16), 4-flúor chalcona (17) e 4-cloro chalcona (18) (Figura 11) apresentaram maior atividade quando comparado com à indometacina (droga padrão). ${ }^{35}$

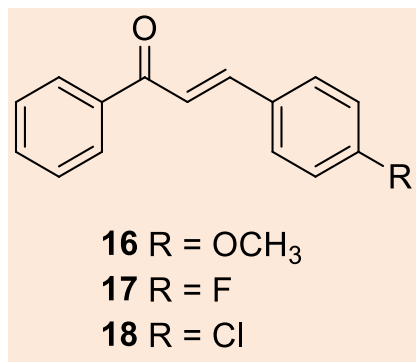

Figura 11. Fórmula estrutural dos derivados da chalcona 3-difenilpropanona

Os derivados de Chalconas exercem efeitos anti-inflamatórios agudos e crônicos, os quais estão relacionados à inibição da superprodução de óxido nítrico (NO), como afirma Alcaraz e colaboradores. ${ }^{36}$ Pesquisas realizadas por Mousa e colaboradores, ${ }^{37}$ comprovaram que os derivados de chalconas cloradas (CHD) (19) e (20) apresentam maior atividade anti-inflamatória devido ao seu efeito na formação de óxido nítrico (Figura 12). Eles apresentaram atividade antiinflamatória quando comparado à dexametasona e foam melhores que o diclofenaco no modelo de granuloma induzido por grânulos de algodão. 


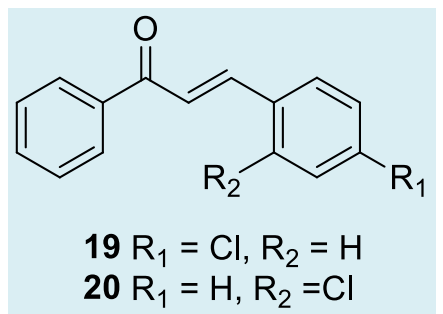

Figura 12. Fórmula estrutural das chalconas cloradas

$\mathrm{Li}$ e colaboradores $^{38}$ sintetizaram e avaliaram a ação biológica de chalconas como agentes anti-inflamatórios. Foram preparadas uma série de 35 novos derivados de chalconas contendo aril-piperazina ou arilsulfonilpiperazina. As atividades dos compostos alvos foram realizadas in vivo e in vitro e avaliadas utilizando o modelo de indução de paraxileno e o ensaio ELISA. Os ensaios anti-inflamatórios in vivo indicaram que a maioria dos compostos alvo apresentaram atividades anti-inflamatórias significativas, especialmente, o composto (21) (Figura 13).<smiles>COc1ccc(/C=C/C(=O)c2ccc(N3CCN(S(=O)(=O)c4ccc(Br)cc4)CC3)cc2)cc1OC</smiles>

Figura 13: Fórmula estrutural do derivado chalcônico com piperazina

\subsection{Atividade anticonvulsivante}

De acordo com o Instituto dos Institutos Nacionais de Saúde (NIH)/NINDS/American Epilepsy Society (AES) Modelos II Workshop, realizado em 2002, o modelo de convulsão "ideal" é aquele que reflete fisiopatologia e fenomenologia semelhantes à convulsão humana. As convulsões devem evoluir espontaneamente após um período latente pós-insucesso ou em um período de tempo de desenvolvimento consistente com a condição humana. O modelo ideal deve apresentar uma abordagem farmacológica que é resistente em pelo menos dois métodos existentes, eletrochoque máximo (MES) e pentilenotetrazol (PTZ).

Esses métodos são os mais utilizados na avaliação da atividade anticonvulsivante, pois fornecem informações sobre a capacidade de um determinado medicamento de penetrar no cérebro e assim exercer um efeito no sistema nervoso central (SNC). ${ }^{39}$ No entanto, estes modelos não são tão seletivos em relação aos mecanismos, mas são adequados para o rastreio da atividade anticonvulsivante, uma vez que nenhum dos dois modelos assume a atividade farmacodinâmica de um medicamento específico. ${ }^{39}$

Com base nas investigações da ação anticonvulsivante de chalconas e derivados sintéticos, pesquisas mostram resultados importantes utilizando os dois métodos. Em estudos apontados por Beyhan e colaboradores, ${ }^{40}$ uma série de chalconas foram preparadas com metil aril cetonas e aldeídos substituídos. Todos os compostos foram testados quanto à sua atividade anticonvulsivante utilizando testes de convulsão induzida por PTZ e MES em camundongos a uma dose de $50 \mathrm{mg} \mathrm{kg}^{-1}$ via 
intraperitoneal. Os resultados da atividade anticonvulsivante demonstraram que entre os compostos testados, os derivados de 2pirazolina-1-carboxamida que transportam 5Bromotiofen-2-il (22) e o 5-Clorotiofen-2-il (23) (Figura 14) exibiram promissora atividade no teste PTZ, uma vez que reduziram a frequência de convulsão em todos os graus (1-
5). Assim, pode-se concluir que os referidos compostos aumentaram o limite convulsivo e prolongaram o tempo de início de 1,20 a 2,10 e 1,20 a 3,58 segundos, assim como aumentaram a taxa de sobrevivência quase duas vezes de $50 \%$ a $95 \%$ e $50 \%$ a $100 \%$, respectivamente.

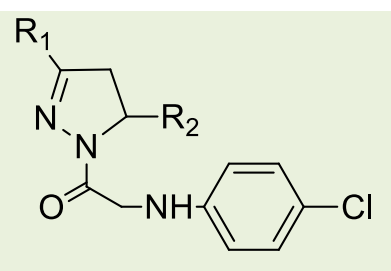

$22 \mathrm{R}_{1}=5$-Bromotiofen-2-il, $\mathrm{R}_{2}=2,6$-Diclorofenil

$23 \mathrm{R}_{1}=5$-Clorotiofen-2-il, $\mathrm{R}_{2}=2,6$-Diclorofenil

Figura 14: Representação estrutural dos derivados de 2-pirazolina-1-carboxamida

A ação anticonvulsivante de chalconas também foi testada em estudo realizado por Genovese e colaboradores ${ }^{41}$ onde sintetizaram alguns preniloxifenilpropanóides naturais e semi-sintéticos (por exemplo, acetofenonas, ácidos benzóico e cinâmico, chalconas e cumarinas). A ação da atividade neuroprotetiva in vivo também foi realizada usando o modelo de convulsão induzido pelo MES. Os compostos 24-27 (Figura 15) protegeram consideravelmente os animais contra convulsões induzidas por MES, possuindo propriedades anticonvulsivas definidas, nos tempos pré-tratamento no modelo MES.<smiles>[R3]CC(C)=CCOc1ccc([R])cc1[R]</smiles>

$24 \mathrm{R}_{1} \mathrm{CH}=\mathrm{CH}-\mathrm{COOH}, \mathrm{R}_{2}=\mathrm{R}_{3}=\mathrm{H}$ $25 \mathrm{CH}=\mathrm{CH}-\mathrm{COOH}, \mathrm{R}_{2}=\mathrm{OCH}_{3}, \mathrm{R}_{3}=$ isopentenil<smiles>[R]CC(C)=CCOc1ccc2c(c1[R2])CC(=O)C=C2</smiles>

$26 \mathrm{R}_{1}=\mathrm{R}_{2}=\mathrm{H}$ $27 \mathrm{R}_{1}=\mathrm{HR}_{2}=\mathrm{OH}$

Figura 15. Fórmula estrutural das preniloxifenilpropanóides

O fato de apenas um número restrito de compostos exibirem uma atividade anticonvulsivante apreciável pode ser justificado pela capacidade desses compostos em interagir com alvos biológicos específicos dentro do SNC, tornando evidente que classes inteiras de compostos, como acetofenonas, derivados de ácido benzóico e chalconas são totalmente inativas. ${ }^{41}$
$O$ método do teste de MES também foi utilizado na investigação de atividade anticonvulsivante de algumas novas fenoxichalconas em pesquisa realizada por Kaushik e colaboradores. Logo, foi observado que os compostos (24) e (25) apresentaram atividade anticonvulsivante significativa sem neurotoxicidade. ${ }^{42}$ 
Estudos recentes realizados por Nassar e colaboradores $^{5}$ investigaram a atividade anticonvulsivante dos derivados de chalcona (28-31) (Figura 16) utilizando o método de MES e teste com PTZ. Todos os compostos sintetizados foram administrados (i.p) em camundongos, utilizando doses de 25, 50, 75 e $100 \mathrm{mg} \mathrm{kg}^{-1}$ como doses de referência, 30 min antes da indução de convulsões e os animais foram observados durante $60 \mathrm{~min}$.
De acordo com os resultados obtidos, a atividade anticonvulsivante das chalconas sintetizadas 28-31 nas doses $30,28,33$ e 37 $\mathrm{mg} \mathrm{kg}^{-1}$, revelaram proteção de $50 \%$ contra convulsões induzidas por PTZ em comparação com as drogas de referência, difenilhidantoína $6,2 \mathrm{mg} \mathrm{kg}^{-1}$, metosuximida $\mathrm{mg} \mathrm{kg}^{-1}$, valproato de sódio $154 \mathrm{mg} \mathrm{kg}^{-1}$, e trimetadiona 260,4 mg $\mathrm{kg}^{-1} .5$

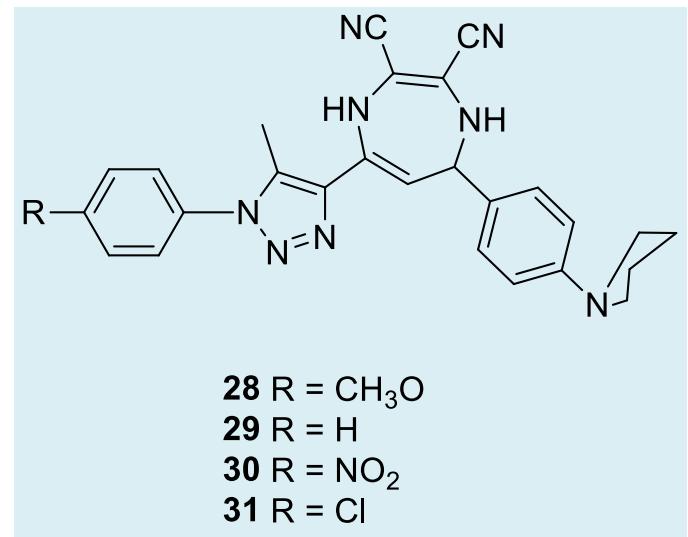

Figura 16: Representação estrutural das chalconas com atividade anticonvulsivante

\subsection{Atividade antiacetilcolinesterase}

A acetilcolinesterase (AChE) é a enzima responsável por hidrolisar o neurotransmissor acetilcolina (ACh) nas sinapses colinérgicas. Nas sinapses, este neurotransmissor (ACh) atua fazendo uma transmissão da mensagem de um neurônio a outro. Como as sinapses estão distribuídas no sistema nervoso central (SNC) e periférico (SNP), este processo é importante para a manutenção de várias funções fisiológicas humanas. ${ }^{43}$ Taylor e colaboradores ${ }^{44}$ ressaltam que existem alguns fármacos que apresentam como alvo exatamente as sinapses colinérgicas, inibindo ou reativando a enzima $\mathrm{AChE}$, atuando ainda em receptores de AChE como agonistas ou antagonistas.

Fármacos que apresentam como mecanismo de ação a inibição da AChE são chamados de anticolinesterásicos ou colinérgicos indiretos. Os fármacos que bloqueiam a AChE no SNP são utilizados na constipação atônica, atonia intestinal, na retenção urinária e como antagonista dos miorrelaxantes. Assim, caso o inibidor da AChE apresente ação no SNC, este por sua vez, possui ação no tratamento da demência associada às doenças de Alzheimer e Parkinson. ${ }^{45,46}$

Sukumaran e colaboradores ${ }^{47}$ sintetizaram 21 hidroxichalconas que demonstraram atividade inibitória da $A C h E$, sendo os compostos mais ativos com valores de $\mathrm{IC}_{50}$ na faixa de 40-85 $\mu \mathrm{M}$. Os valores de $\mathrm{IC}_{50}$ dos quatro compostos com maior inibição de AChE também foram determinados para Butirilcolinesterase (BChE) equino, considerado um bom modelo para o $\mathrm{BChE}$ humano, ${ }^{48}$ uma vez que a forma de equino tem $90 \%$ de identidade de sequência em comparação com a forma humana. Todos os compostos mostraram atividade inibitória BChE apreciável, mas geralmente em níveis mais baixos do que para AChE. 
Ao examinar o efeito da substituição no anel B, as chalconas 32-35 (Figura 17) com substituintes halogênios $(\mathrm{Br} / \mathrm{Cl})$ mostraram a maior atividade (38 $\mu \mathrm{M} ; 30 \mu \mathrm{M} ; 23 \mu \mathrm{M}$ e 51 $\mu \mathrm{M}$, respectivamente) embora nem todos os compostos com substituintes halogênios no anel B tenham apresentado atividades mensuráveis a $10 \mu \mathrm{M}$.<smiles>[R]c1cc([R5])c(C(=O)/C=C/c2cc([R4])c([R3])c([R])c2[R])c(O)c1</smiles>

$32 \mathrm{R}_{1}=\mathrm{Br}, \mathrm{R}_{2}=\mathrm{R}_{3}=\mathrm{R}_{4}=\mathrm{R}_{5}=\mathrm{R}_{6}=\mathrm{H}$

$33 \mathrm{R}_{1}=\mathrm{H}, \mathrm{R}_{2}=\mathrm{H}, \mathrm{R}_{3}=\mathrm{Br}, \mathrm{R}_{4}=\mathrm{H}, \mathrm{R}_{5}=\mathrm{OCH}_{3}, \mathrm{R}_{6}=\mathrm{OCH}_{3}$

$34 \mathrm{R}_{1}=\mathrm{Br}, \mathrm{R}_{2}=\mathrm{H}, \mathrm{R}_{3}=\mathrm{R}_{4}=\mathrm{H}, \mathrm{R}_{5}=\mathrm{OCH}_{3}, \mathrm{R}_{6}=\mathrm{OCH}_{3}$

$35 \mathrm{R}_{1}=\mathrm{OH}, \mathrm{R}_{2}=\mathrm{Cl}, \mathrm{R}_{3}=\mathrm{H}, \mathrm{R}_{4}=\mathrm{Cl}, \mathrm{R}_{5}=\mathrm{OCH}_{3}, \mathrm{R}_{6}=\mathrm{OCH}_{3}$

Figura 17. Fórmula estrutural das hidroxichalconas

Os autores concluíram que os compostos com substituintes metoxi no anel $A$ e os substituintes halogênios no anel B foram geralmente considerados com atividade mais potente quando comparados com o propídio, um inibidor padrão de AChE.

Liu e colaboradores $^{49}$ investigaram os efeitos inibitórios de chalconas com diferentes cadeias laterais ( $n=2-6$ ) (Figura 18) em AChE e BuChE utilizando o método Ellman e Rivastigmina como o controle positivo.

De acordo com os dados, todos os compostos sintetizados apresentaram maiores atividades inibitórias contra AChE do que o composto precursor $\left(I \mathrm{C}_{50}>500 \mathrm{~mol} \mathrm{~L}^{-1}\right)$. Portanto, os compostos $36\left(\mathrm{IC}_{50}=4,68 \mathrm{~mol} \mathrm{~L}^{-}\right.$ $\left.{ }^{1}\right), 37\left(\mathrm{IC}_{50}=7,63 \mathrm{~mol} \mathrm{~L}^{-1}\right), \mathbf{3 8}\left(\mathrm{IC}_{50}=8,95 \mathrm{~mol} \mathrm{~L}^{-}\right.$ $\left.{ }^{1}\right)$ e $39\left(I_{50}=5,91 \mathrm{~mol} \mathrm{~L}^{-1}\right)$ (Figura 18) apresentaram potentes atividades em comparação com o composto de referência Rivastigmina $\left(I C_{50}=10,54 \mathrm{~mol} \mathrm{~L}^{-1}\right)$.

A chalcona 36 foi considerada a mais promissora, uma vez que esta foi 2 vezes mais ativa do que Rivastigmina e também apresentou alta seletividade para AChE sobre BuChE. Diante disso, os autores enfatizam que a variação do comprimento da cadeia que liga o esqueleto da chalcona com grupos de aminas terminais influenciou significativamente a sua atividade inibidora. Com base no estudo cinético realizado, os compostos variaram entre 1,49 e 2,19, o que indicou que todos os compostos eram possivelmente suficientemente lipofílicos para passar BBB in vivo. ${ }^{49}$

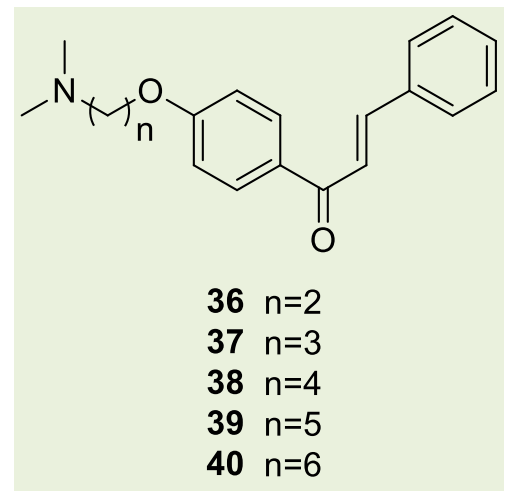

Figura 18. Fórmula estrutural das chalconas derivadas da 2-hidroxiacetofenona 
Tran e colaboradores ${ }^{50}$ realizaram o teste de inibição de uma nova série de derivados de benzilaminochalcona (Figura 19) com diferentes substituintes no anel B. Os pesquisadores utilizaram também o método de Ellman. Os resultados mostraram que a maioria dos compostos estudados apresentaram atividade de inibição de AChE fracas a moderadas. Dessa forma, observaram que os substituintes 4-clorofenil (41), 4nitrofenil (42), 3,4-dimetoxifenil (43) e piridin4-il (44) presentes no anel B apresentaram atividades inibitórias significativas contra a enzima $A C h E$, com valores de $\mathrm{IC}_{50}=23,71$; 31,$57 ; 23,02$ e $38,97 \mu \mathrm{M}$, respectivamente.<smiles>[R]c1ccc(/C=C/C(=O)c2ccc(NCc3ccccc3O)cc2)cc1[R]</smiles>

$41 \mathrm{R} 1=\mathrm{Cl}, \mathrm{R}_{2}=\mathrm{H}$ $42 \mathrm{R} 1=\mathrm{NO}_{2}, \mathrm{R}_{2}=\mathrm{H}$ $43 \mathrm{R}_{1}=\mathrm{R}_{2}=\mathrm{OCH}_{3}$<smiles>O=C(/C=C/c1ccncc1)c1ccc(NCc2ccccc2O)cc1</smiles>

44

Figura 19. Representação estrutural das benzilaminochalconas

Os estudos de modelagem molecular desta pesquisa foram consistentes com a hipótese de que as benzilaminochalconas possam exercer seus efeitos como inibidores da AChE no sítio de ligação dupla, que podem simultaneamente aumentar a neurotransmissão colinérgica e inibir a agregação de amiloides através da ligação tanto catalítica como em sítios periféricos da enzima. Assim, esses derivados poderiam ser desenvolvidos para fornecer novas pistas para a descoberta de novas drogas antialzheimer. ${ }^{50}$

\section{Considerações Finais}

Chalconas são uma classe de flavonoides que estão presentes em produtos regularmente utilizados no consumo humano como: frutas, vegetais, grãos, raízes, flores, chás e vinhos, podem ser obtidas de fontes naturais ou sintetizadas e, são compostos de grande interesse químico-farmacológico devido principalmente a sua estrutura relativamente simples e a diversidade de atividades farmacológicas que apresentam, dentre as quais podemos destacar: atividade antioxidante, antinociceptiva, anticonvulsivante e anti-inflamatória. Portanto, as informações apresentadas nesse manuscrito podem ser de grande interesse para futuras investigações sobre o aspecto químico e farmacológico de chalconas.

\section{Agradecimentos}

A FUNCAP e CNPq pelo apoio financeiro, ao Mestrado Acadêmico em Recursos Naturais da UECE. A Universidade Estadual Vale do Acaraú e ao Governo do Estado do Ceará.

\section{Referências Bibliográficas}

${ }^{1}$ Kuete, V.; Mbaveng, A. T.; Zeino, M.; Fozing, C. D.; Ngameni, B.; Kapche, G. D. W. F.; Ngadjui, B. T.; Efferth, T. Cytotoxicity of three naturally occurring flavonoid derived compounds (artocarpesin, cycloartocarpesin and isobavachalcone) towards multi-factorial drug-resistant cancer cells. Phytomedicine 2015, 22, 1096. [CrossRef] [PubMed] 
${ }^{2}$ Lima, D. C. S.; do Vale, C. R.; Véras, J. H.; Bernardes, A.; Pérez, C. N.; Chen-Chen, L. Absence of genotoxic effects of the chalcone (E)-1-(2-hydroxyphenyl)-3-(4-methylphenyl)prop-2-en-1-one) and its potential chemoprevention against DNA damage using in vitro and in vivo assays. Plos one 2017, 12, e0171224. [CrossRef] [PubMed]

${ }^{3}$ Vanangamudi, G.; Subramanian, M.; Thirunarayanan, G. Synthesis. Spectral linearity, antimicrobial, antioxidant and insect antifeedant activities of some 2,5-dimethyl-3thienyl chalcones. Arabian Journal of Chemistry 2017, 10, S1254. [CrossRef]

${ }^{4}$ Mohamad, A. S.; Akhtar. M. N.; Zakaria, Z. A.; Perimal, E. K.; Khalid, S.; Mohd, P.A.; Khalid, M. H.; Israf, D. A.; Lajis, N. H.; Sulaiman, M. R. Antinociceptive activity of a synthetic chalcone, flavokawin B on chemical and thermal models of nociception in mice. European Journal of Pharmacology 2010, 647, 103. [CrossRef] [PubMed]

${ }^{5}$ Nassar, E. M.; Abdelrazek, F. M.; Ayyad, R. R.; El-Farargy, A. F. Synthesis and Some Reactions of 1-aryl-4-acetyl-5-methyl-1,2,3-triazole Derivatives with Anticonvulsant Activity. Mini reviews in medicinal chemistry 2016, 16, 926. [CrossRef] [PubMed]

${ }^{6}$ Özdemir, A.; Altıntop, M. D.; Turan-Zitouni, G.; Çiftçi, G. A.; Ertorun, I.; Alataş, Ö.; Kaplanciclı, Z. A. Synthesis and Evaluation of New Indole-Based Chalcones as Potential Antiinflammatory Agents. European Journal of Medicinal Chemistry 2015, 89, 304. [CrossRef] [PubMed]

${ }^{7}$ Ahmad, S.; Israf, D. A.; Lajis, N. H.; Shaari, K.; Mohamed, H.; Wahab, A. A.; Ariffin, K. T.; Hoo, W. Y.; Aziz, N. A.; Kadir, A. A.; Sulaiman, M. R.; Somchit, M. N. Cardamonin, inhibits proinflammatory mediators in activated RAW 264.7 cells and whole blood. European Journal of Pharmacology 2006, 538, 188. [CrossRef] [PubMed]

${ }^{8}$ Mora-Pérez, A.; Hernández-Medel, M. R. Anticonvulsant activity of methanolic extract from Kalanchoe pinnata (Lam.) stems and roots in mice: A comparison to diazepam.
Neurología 2016, 31, 161. [CrossRef] [PubMed]

${ }^{9}$ Nijveldt, R. J.; van Nood, E.; van Hoorn, D. E. C; Boelens, P. G.; van Norren, K.; van Leeuwen, P. A. M. Flavonoids: a review of probable mechanisms of action and potential applications. The American Journal of Clinical Nutrition 2001, 74, 418. [CrossRef] [PubMed]

${ }^{10}$ Martens, S.; Mithofer, A. Flavones and flavone synthases. Phytochemistry 2005, 66, 2399. [CrossRef]

${ }^{11}$ Treutter, D. Significance of Flavonoids in Plant Resistance and Enhancement of Their Biosynthesis. Plant Biology 2005, 7, 581. [CrossRef] [PubMed]

12 Nowakowska, Z. A review of anti-infective and anti-inflammatory chalcones. European Journal of Medicinal Chemistry 2007, 42, 125. [CrossRef] [PubMed]

${ }^{13} \mathrm{Ni}$, L.; Meng, C. Q.; Sikorski, J. A. Recent advances in therapeutic chalcones. Expert Opinion on Therapeutic Patents 2004, 14, 1669. [CrossRef]

${ }^{14}$ Shimokoriyama, M. Em Flavanones chalcones and aurones. Geissman, T. A. ed.; MacMillan Company: New York:, 1962, cap. 3.

${ }^{15}$ Iwashina T. The structure and distribution of the flavonoids in plants. Journal of Plant Research 2000, 113, 287. [CrossRef]

${ }^{16}$ Narender, T.; Venkateswarlu, K.; Nayak, B. V.; Sarkar, S. A new chemical access for 3'acetyl-4'-hydroxychalcones using borontrifluoride-etherate via a regioselective Claisen-Schmidt condensation and its application in the synthesis of chalcone hybrids. Tetrahedron Letters 2011, 52, 5794. [CrossRef]

${ }^{17}$ Shang, Y. F.; Oidovsambuu, S.; Jeon, J.-S.; Nho, C. W.; Um, B.-H. Chalcones from the flowers of Coreopsis lanceolata and their in vitro antioxidative activity. Planta Medica 2013, 79, 295. [CrossRef] [PubMed]

${ }^{18}$ Abegaz, B. M.; Ngadjuib, B. T.; Dongo, E.; Ngameni, B.; Nindi, M. N.; Bezabiha, M. Chalcones and other constituents of Dorstenia prorepens and Dorstenia zenkeri. 
Phytochemistry 2002, 59, 877. [CrossRef] [PubMed]

${ }^{19}$ Jayasinghe, L.; Balasooriya, B. A. I. S.; Padmini, W. C.; Hara, N.; Fujimoto, Y. Geranyl chalcone derivatives with antifungal and radical scavenging properties from the leaves of Artocarpus nobilis. Phytochemistry 2004, 65, 1287. [CrossRef] [PubMed]

${ }^{20}$ Yang, Y.; Zhang, T.; Xiao, L.; Yang, L.; Chen, $R$. Two new chalcones from leaves of Morus alba L. Fitoterapia 2010, 81, 614. [CrossRef] [PubMed]

${ }^{21}$ Li, Y.-P.; Yang, Y.-C.; Li, Y.-K.; Jiang, Z.-Y.; Huang, X.-Z.; Wang, W.-G.; Gao, X.-M.; Hu, Q.$F$. Prenylated chalcones from Desmodium renifolium. Phytochemistry Letters 2014, 9, 41. [CrossRef]

${ }^{22}$ Nishimura, R.; Tabata, K.; Arakawa, M.; Ito, Y.; Kimura, Y.; Akihisa, T.; Nagai, H.; Sakuma, A.; Kohno, H.; Suzuki, T. Isobavachalcone, a chalcone constituent of Angelica keiskei, induces apoptosis in neuroblastoma. Biological and Pharmaceutical Bulletin 2007, 30, 1878. [CrossRef] [PubMed]

${ }^{23}$ Pandey, M. M.; Khatoon, S.; Rastogi, S.; Rawat, A. K. S. Determination of flavonoids, polyphenols and antioxidant activity of Tephrosia purpurea: a seasonal study. Journal of Integrative Medicine 2016, 14, 447. [CrossRef] [PubMed]

${ }^{24}$ Lahsasni, S. A.; Al Korbi, F. H.; Aljaber, N. A.A. Synthesis, characterization and evaluation of antioxidant activities of some novel chalcones analogues. Chemistry Central Journal 2014, 8, 32. [CrossRef]

${ }^{25}$ Cheng, Z.-J.; Lin, C.-N.; Hwang, T.-L.; Teng, C.-M. Broussochalcone $A$, a potent antioxidant and effective suppressor of inducible nitric oxide synthase in lipopolysaccharideactivated macrophages. Biochemical Pharmacology 2001, 61, 939. [CrossRef] [PubMed]

${ }^{26}$ Shenvi, S.; Kumar, K.; Hatti, K. S.; Rijesh, K.; Diwakar, L.; Reddy, G. C. Synthesis, anticancer and antioxidant activities of 2, 4, 5-trimethoxy chalcones and analogues from asaronaldehyde: Structure-activity relationship. European Journal of Medicinal Chemistry 2013, 62, 435. [CrossRef] [PubMed]

${ }^{27}$ Prabhakar, V.; Iqbal, H.; Balasubramanian, R. Antioxidant studies on monosubstituted chalcone derivatives - understanding substituent effects. Pakistan Journal of Pharmaceutical Sciences 2016, 29, 165. [Link] [PubMed]

${ }^{28}$ Barrot, M. Test and models of nociception and pain in rodents. Neuroscience 2012, 211, 39. [CrossRef] [PubMed]

${ }^{29}$ Millan, M. J. Descending control of pain. Progress in Neurobiology 2002, 66, 355. [CrossRef]

${ }^{30}$ Heidari, M. R.; Foroumadi, A.; Amirabadi, A.; Samzadeh-Kermani, A.; Azimzadeh, B. S.; Eskandarizadeh, A. Evaluation of antiinflammatory and analgesic activity of a novel rigid 3,4-dihydroxy chalcone in mice. Annals of the New York academy of sciences 2009, 1171, 399. [CrossRef] [PubMed]

${ }^{31}$ Ismail, N. I.; Ming-Tatt, L.; Lajis, N.; Akhtar, M. N.; Akira, A.; Perimal, E. K.; Israf, D. A.; Sulaiman, M. R. Antinociceptive Effect of 3(2,3-Dimethoxyphenyl)-1(5-methylfuran-2-

$\mathrm{yl})$ prop-2-en-1-one in Mice Models of Induced Nociception. Molecules 2016, 21, 1077. [CrossRef]

${ }^{32}$ Corrêa, R.; Pereira, M. A. S.; Buffon, D.; dos Santos, L.; Cechinel Filho, V.; Santos, A. R. S.; Nunes, R. J. Antinociceptive properties of chalcones. Structure-activity relationships. Archiv der Pharmazie Weinheim 2001, 334, 332. [CrossRef] [PubMed]

${ }^{33}$ Geetha, T.; Varalakshmi, P. Antiinflammatory activity of lupeol and lupeol linoleate in rats. Journal of Ethnopharmacology 2001, 76, 77. [CrossRef] [PubMed]

${ }^{34}$ Viana, G. S. B.; Bandeira, M. A. M.; Matos, F. J. A. Analgesic and antiinflammatory effects of chalcones isolated from Myracrodruon urundeuva Allemão. Phytomedicine 2003, 10, 189. [CrossRef] [PubMed]

${ }^{35}$ Botelho, M. A.; Rao, V. S.; Montenegro, D.; Bandeira, M. A. M.; Fonseca, S. G. C.; 
Nogueira, N. A. P.; Ribeiro, R. A.; Brito, G. A. C. Effects of a herbal gel containing carvacrol and chalcones on alveolar bone resorption in rats on experimental periodontitis. Phytotherapy Research 2008, 22, 442. [CrossRef] [PubMed]

${ }^{36}$ Alcaraz, M. J.; Fernández, P.; Guillén, M. I. Anti-inflammatory actions of the heme oxygenase-1 pathway. Current Pharmaceutical Design 2003, 9, 2541. [CrossRef] [PubMed]

${ }^{37}$ Mousa, M. N.; Muhasin, R. J.; Alrubaie, L. A. R. Synthesis and Assessment of Antiinflammatory Activity of a Chlorosubstituted Chalcone Derivatives and using the Semiempirical Methods to Measure the Linked Physicochemical Parameters. Journal of Pharmaceutical and Biomedical Sciences 2016, 6, 583. [Link]

${ }^{38}$ Li, J.; Li, D.; Xu, Y.; Guo, Z.; Liu, X.; Yang, H.; $\mathrm{Wu}, \mathrm{L}$.; Wang, L. Design, synthesis, biological evaluation, and molecular docking of chalcone derivatives as anti-inflammatory agents. Bioorganic \& Medicinal Chemistry Letters 2017, 27, 602. [CrossRef] [PubMed]

${ }^{39}$ Smith, M.; Wilcox, K. S.; White H. S. Discovery of Antiepileptic Drugs. Neurotherapeutics 2007, 4, 17. [CrossRef]

${ }^{40}$ Beyhan, N.; Kocyigit-kaymakcioglu, B. Synthesis and anticonvulsant activity of some 2-pyrazolines derived from chalcones. Arabian Journal of Chemistry 2017, 10, S2073. [CrossRef]

${ }^{41}$ Genovese, S.; Epifano, F.; Curini, M.; Dudrajastrzebska, M.; Luszczki, J. J. Prenyloxyphenylpropanoids as a novel class of anticonvulsive agents. Bioorganic \& Medicinal Chemistry Letters 2009, 19, 5419. [CrossRef] [PubMed]

${ }^{42}$ Kaushik, S.; Kumar, N.; Drabu, S. Synthesis and anticonvulsant activities of phenoxychalcones. The Pharma Research 2010, 3, 257. [Link]

4343 Westfall, T. C.; Westfall, D. P. Em Goodman \& Gilman. As bases farmacológicas da terapêutica, 10a. Ed.; Brunton, L. L.; Lazo, J. S.; Parker, K. L., eds.; Mc Graw Hill: Rio de Janeiro, 2006, cap. 6.

${ }^{44}$ Taylor, P. Em Goodman \& Gilman. As bases farmacológicas da terapêutica, 10a. Ed.; Brunton, L. L.; Lazo, J. S.; Parker, K. L., eds.; Mc Graw Hill: Rio de Janeiro, 2006, cap. 8.

45 Inouye, K.; de Oliveira, G. H. Avaliação crítica do tratamento farmacológico atual para Doença de Alzheimer. Infarma Ciências farmacêuticas 2004, 15, 80. [LinK]

${ }^{46}$ Standaert, D. G.; Young, A. B. Em Goodman \& Gilman. As bases farmacológicas da terapêutica, 10a. Ed.; Brunton, L. L.; Lazo, J. S.; Parker, K. L., eds.; Rio de Janeiro Mc Graw Hill: Rio de Janeiro, cap. 20.

${ }^{47}$ Sukumaran, S. D.; Chee, C. F.; Viswanathan, G.; Buckle, M. J. C.; Othman, R.; Rahman, N. A.; Chung, L. Y. Synthesis, Biological Evaluation and Molecular Modelling of 2'Hydroxychalcones as Acetylcholinesterase Inhibitors. Molecules 2016, 21, 955. [CrossRef] [PubMed]

${ }^{48}$ Sawatzky, E.; Wehle, S.; Kling, B.; Wendrich, J.; Bringmann, G.; Sotriffer, C. A.; Heilmann, J.; Decker, M. Discovery of highly selective and nanomolar carbamate-based butyrylcholinesterase inhibitors by rational investigation into their inhibition mode. Journal of Medicinal Chemistry 2016, 59, 2067. [CrossRef] [PubMed]

${ }^{49}$ Liu, H.-R.; Liu X.-J.; Fan, H.-Q.; Tang, J.-J.; Gao, X.-H.; Liu, W.-K. Design, synthesis and pharmacological evaluation of chalcone derivatives as acetylcholinesterase inhibitors. Bioorganic \& Medicinal Chemistry 2014, 22, 6124. [CrossRef]

${ }^{50}$ Tran, T.-D.; Nguyen, T.-C.-V.; Nguyen, N.-S.; Nguyen, D.-M.; Nguyen, T.-T.-H; Le, M.-T.; Thai, K.-M. Synthesis of Novel Chalcones as Acetylcholinesterase Inhibitors. Applied Sciences 2016, 6, 198. [CrossRef] 